\title{
Development and behavioural toxicity of deltamethrin on Rattus norvegicus following gestational exposure
}

\author{
Joya and G.K. Sangha* \\ Department of Zoology, Punjab Agricultural University, Ludhiana-141004 (Punjab), INDIA \\ *Corresponding author. E-mail:sanghagk@hotmail.com \\ Received: February 28, 2015; Revised received: September 19, 2015; Accepted: January 16, 2016
}

\begin{abstract}
Deltamethrin, a synthetic type II pyrethroid was used at acceptable daily intake (ADI) levels to evaluate the embryo toxic risk in rat offsprings. Commercial formulation of deltamethrin (Decis 2.8 EC) at the level of ADI $(0.01 \mathrm{mg} / \mathrm{kg} \mathrm{bw} /$ day) and ten times ADI $(0.1 \mathrm{mg} / \mathrm{kg}$ bw/day) was given orally to female rats (Rattus norvegicus) after mating until the end of pregnancy. The rats which did not breed were sacrificed for uterine examination, total implantations and resorptions. No toxicological symptoms were found in the rats treated with ADI level of deltamethrin, however rats treated with 10 times ADI dose of deltamethrin appeared weak and less active. Rats treated with 10xADI did not breed and resorptions and cysts were observed in their uterus and ovary respectively. In rats treated with $\mathrm{ADI}$ dose of deltamethrin, litter size was reduced, pups gained less body weight and their developmental parameters and behavioural milestones were delayed as compared to control rats pups. The results suggested that gestational exposure of deltamethrin on growth and viability of rat offsprings at ADI level also affected the development and behaviour of rat offsprings.
\end{abstract}

Keywords: Behaviour, Deltamethrin, Development, Female rats, Rat pups

\section{INTRODUCTION}

The indiscriminate and injudicious use of pesticides poses threat to human health as pesticide residue can enter food chain and when their concentration exceeds the maximum limit, ill effects are produced (McLachlan, 2001). Humans are increasingly exposed to pesticides at all stages of life due to changed lifestyle, including when the babies are still at mother's womb (Xavier et al 2004). Epidemiological studies have documented an association between spontaneous abortions, foetal death after maternal exposure of pesticides (Wigle et al., 2008).

Synthetic pyrethroid insecticides are now being substituted for pest control and increased production (Muthviveganandavel et al., 2008). Exposure to pyrethroids has been widely documented in pregnant woman, infants and children (Schettgen et al., 2002; Berkowitz et al 2003). Pyrethroids increase embryonic resorption and fetal mortality (Yousef et al., 2003) and fetotoxicity (Ahmad and Khan, 2012). Maternal exposure to cypermethrin affected the body and organ weight of offsprings (Huang and $\mathrm{Li}, 2014$ ). Decrease in the implantation sites, corpora lutea and recovered foetuses from uterine horns and increased incidence of pre- and post-implantation losses and early mortality rate in pyrethroid exposed animals have also been reported (Shukla and Taneja, 2002; Ullah et al., 2006; Ahmad, 2010).

Deltamethrin, a synthetic pyrethroid with potential insecticidal property is extensively used as an ectoparasiticide in crop protection and public health programme (McGregor, 2000). Deltamethrin administered during period of major organogenesis reduced the average weight of the live foetuses (Bhaumik and Gupta, 1990). The incidence of early embryonic deaths by causing retardation of growth, hypoplasia of the lungs and increase in placental weight was higher in deltamethrin treated rats than in control animals (Abdel-Khalik et al., 1993). Deltamethrin (2mg/kg bw) treatment in wistar rats from pregnancy through lactation pose reproductive hazards by increasing the estrogenic potency of target organs (Presibella et al., 2005). Information regarding maternal deltamethrin effect at ADI level on development and behavior of rat offsprings is lacking. Therefore, the present investigation was conducted to study the development and behavioural toxicity of deltamethrin on rats (rattus norvegicus) following gestational exposure.

\section{MATERIALS AND METHODS}

Animals: Sexually mature female albino rats weighting 100-150 gm were procured from Department of Livestock Production and Management, Guru Angad Dev Veterinary and Animal Sciences University, Ludhiana. The rats were acclimatized for one week before starting the treatment. The experiments were carried out according to the national regulations and rules for animal welfare and guidelines of Institutional Animal Ethical Committee (IAEC), Guru Angad Dev 
Veterinary and Animal Sciences University, Ludhiana. Experimental designAdult female rats with normal cyclicity were mated with young males previously determined to be fertile in the ratio of $3: 1$ and kept together until fertilization is proved either by observing vaginal plug or presence of sperms in the vaginal smears. It was designated as gestation day 0 (GD0). Deltamethrin (Decis 2.8 EC from M/S Bharat Pesticide Limited, Mumbai) at the level of ADI $(0.01 \mathrm{mg} / \mathrm{kg} \mathrm{bw} /$ day) and ten times ADI $(0.1 \mathrm{mg} / \mathrm{kg} \mathrm{bw} /$ day $)$ was given to female rats after mating until the end of pregnancy by oral intubation. Adequate dilutions were made with peanut oil to achieve test concentrations. The test concentrations were calculated from the percentage of active ingredients of commercial formulations. Control rats also received the similar amount of peanut oil (without any pesticide) orally through intubation.

Toxicological symptoms: All the pregnant rats were observed daily (control and treated) for toxicological symptoms. The pregnant rats were weighed at weekly intervals till parturition. The rats are allowed to complete their gestation period. At the end of gestation period the females were allowed to produce litter normally. The rats which did not breed were sacrificed for uterine examination. Total implantations, resorptions, number of live and dead fetuses were observed in these rats. The foetuses were weighed and examined for macroscopic external malformations.

Developmental and behavioural milestones in offsprings: Gestation length was calculated at birth and on Postnatal Day 1 (PND1) sex ratio (as percent males), body weight for each pup and the number of malformed offsprings was noted. Pups remained with their biological mothers and all the viable pups were weighed on PND1, PND7, PND14, PND21, PND28, PND35 and PND42 following birth for recording growth and survival in rats. Physical parameters of development like general behaviour were assessed daily. Pinna detachment, incisor eruption, eyes opening, fur eruption and ear folding, were also observed as described by Gandhi et al (2014).

The dams were removed from home cage and individual tests within each testing category were preceded in following order:

For righting reflex, the pup was placed on its back (dorsal position) and the time required (latency(s)) for the animal to turn ventrally itself within $60 \mathrm{sec}$ was recorded. Other reflexes such as palmer grasps(PND11), negative geotaxis (PND 9-11), cliff avoidance (PND-4), free-fall righting (PND 12-14), forelimb placing (PND4 -5) and hind limb placing (PND6) were assessed as described by (Altman and Sudarshan, 1975, Lapointe and Nosal, 1979). Palmer grasp as evaluated by stroking gently the palm of forepaw with a forceps and observing the digital flexing response.

\section{RESULTS AND DISCUSSION}

All the control rats were normal, healthy and active during the treatment period. No toxicological symptoms were found in the rats treated with ADI level of deltamethrin. However, rats treated with 10 times ADI dose of deltamethrin appeared weak and less active. Earlier studies of Kumar and Nagar (2014) in deltamethrin treated rats also did not show any significant changes in behaviour and appearance. Signs of lethargy were also observed in female rats treated with three doses of deltamethrin $(5.35,13.38$ and $26.75 \mathrm{mg} / \mathrm{kg}$ body weight) during the gestation period (Kandil, 2006).

Body weight: The rats treated with ADI dose of deltamethrin showed more body weight gain in $\mathrm{I}^{\text {st }}$ week but there after the gain was less as compared to control rats, In the third week of pregnancy the body weight gain was non significantly less in ADI dose pregnant females. Females treated with 10 times ADI dose deltamethrin showed significantly minimal body weight gain during four weeks of study (Fig. 1).

A decrease in maternal body weight gain during gestation was also noted by Kandil (2006) in deltamethrin treated rats. Dams treated with fenitrothion showed signs of toxicity in two highest dose groups (20 and 25 $\mathrm{mg} / \mathrm{kg} /$ day), as evidenced by a significant decrease (14\% and $19 \%$, respectively) in body weight gain between gestational day 12 and 21 (Turner et al 2002).

Reproductive outcome: The percent pregnancy of control rats was $100 \%$, and in rats treated with ADI of deltamethrin the percent pregnancy was $50 \%$. None of the rats treated with 10 times of ADI of deltamethrin became pregnant (Table 1). The gestational length was prolonged significantly in deltamethrin treated rats. In control rats the gestational length ranged 23-24 days while in rats treated with ADI level of deltamethrin it was prolonged to 27-29 days (Table 1). Exposure to $1.0 \mathrm{mg} / \mathrm{kg} /$ day of $\mathrm{MeHg}$ (methyl mercury) treatment group also showed prolonged gestation period (Gandhi et al 2014). However, increased exposure to malathion, parathion and chlorpyrifos have results in reduced gestational length (Eskenazi et al., 2004). Triazophos at a dose level $1 / 10^{\text {th }}$ of $\mathrm{LD}_{50}$ have also resulted in slight reduction in gestational length of female rats (Sharma and Sangha, 2014).

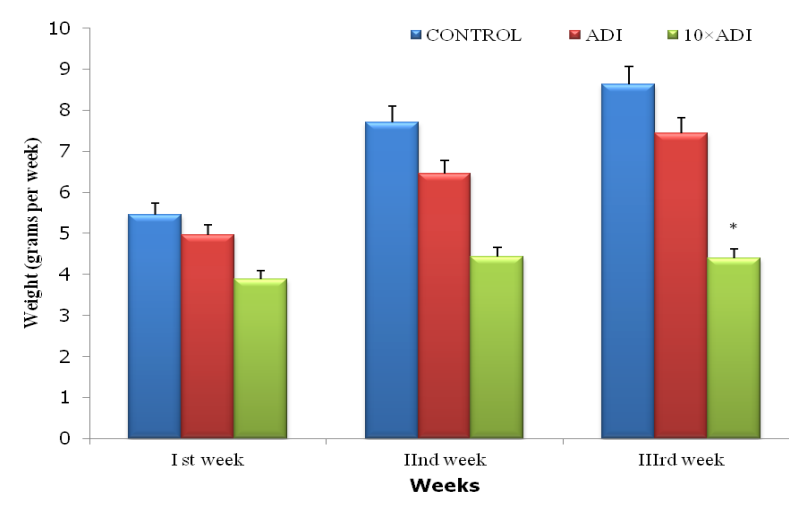

Fig. 1. Weekly changes in body weight gain (g/week) in deltamethrin $(A D I$ and $10 \times A D I)$ treated female albino rats and control rats during pregnancy. 
In control rats the litter size ranged from 7-8 pups per animal. Significantly reduced litter size was observed in rats treated with ADI level of deltamethrin and only 1-4 pups were born to ADI treated rats (Table 1). All the pups were alive in control rats while there was $20 \%$ mortality in pups born to treated rats with ADI of deltamethrin (Table 1). It was found that more females were born to ADI of deltamethrin treated mothers as compared to control mothers where the male female ratio was $1.1: 1$.

In utero exposure is widely considered to be the most sensitive exposure time in terms of reproductive effects (Mocarelli et al., 2000). The mammalian hormone levels around the time of conception are associated with the sex of resulting offspring. The male to female ratio at birth is a marker of parental endocrine disruption (James, 2008). A decreased male to female ratio may arise from over expression of aromatase ( $\mathrm{Li}$ et al., 2001; Li and Rehman, 2008). Huang and Li (2014) also found that sex ratio of offspring was decreased significantly in dose dependent manner in cypermethrin treated rats. Parental exposure to both dioxin and vinclozolin has been shown to cause excess female offspring due to altered hormone conception (Zober et al., 1995, Mocarelli et al., 2000). The exposure of pregnant animals to pyrethroids decreased fertility and produced foetal mortality (Ahmad, 2010). Fenitrothion exposure also induced fetotoxicity, as evidenced by an increased incidence of fetal death. Treatment with permethrin $(9.8$ and $19.6 \mathrm{mg} / \mathrm{kg} / \mathrm{day}$, for 4 weeks) also significantly decreased litter size, pup survival and pup growth in progeny of treated female rats mated with treated males (Farag et al., 2007). A significant decrease in pregnancy rate, number of implantation sites and total number of recovered fetuses have also been reported in female animals receiving pyrethroid treatment during gestation (Ahmad and Khan, 2012).

Ovarian cysts were observed in rats treated with $10 \times \mathrm{ADI}$ of deltamethrin. More resorption sites as well tumour in uterus and ovary of $10 \times \mathrm{ADI}$ of deltamethrin treated rats as compared to ADI of deltamethrin treated rats were also observed. Exposure to pesticides has been implicated in the aetiologies of miscarriage and other reproductive disorders (Garry, 2004). The number of pups born alive following late gestational exposure to 20 and $25 \mathrm{mg}$ fenitrothion/ $\mathrm{kg} /$ day was decreased significantly, even though the number of implantation sites in these two exposed groups similar to that found in control litters. This implies that pesticides induce post implantation losses and decreased fetal viability (Turner et al 2002). In present study also decreased litter size, pup survival and pup growth in treated rats as compared to control rats was observed. A dose dependent and significantly decreased number of foetuses vs. number of CL (embryonic resorption) and dose dependently increased foetal mortality along with delayed ossification of bones have been documented when cypermethrin was administered in female rabbits during gestation (Biernacki et al., 1995; Ullah et al., 2006).

Decrease in the implantation sites, corpora lutea and recovered fetuses from uterine horns and increased incidence of pre- and post-implantation losses and early mortality rate in pyrethroid exposed animals have been reported (Shukla and Taneja, 2002; Ullah et al., 2006; Ahmad, 2010). Sublethal doses of both XenTari® and deltamethrin produced qualitative/ quantitative alterations in the blastocyst-endometrium interaction in female rats, thereby compromising the implantation process (Lemos et al., 2011).

Evaluation of progeny: It was observed that there was a significant decrease in weight gain of pups born to ADI deltamethrin treated mothers during $\mathrm{I}^{\mathrm{st}}, \mathrm{II}^{\mathrm{nd}}, \mathrm{VI}^{\text {th }}$ week and VII ${ }^{\text {th }}$ week as compared to control pups (Table 2).

Ahmed and Gupta (1988) have also observed that, cypermethrin when given orally to female rats resulted in significant reduced body weight gain in pups at different days of lactation. Husain and Seth (1991) reported that when deltamethrin was administered by gavage at $7 \mathrm{mg} / \mathrm{kg} /$ day to pregnant rats on gestation days 5 to 21 and to post-weaning rats on postnatal days 22 to 37 , the treated pups during gestation had reduced birth weight and growth rate. Low birth weight and foetal growth outcomes were also observed in pesticides

Table 1. Effect of preconceptional exposure of deltamethrin on reproductive parameters of dams.

\begin{tabular}{lccc}
\hline Parameters & Control & ADI of Deltamethrin & 10×ADI of Deltamethrin \\
\hline Number of Females paired & 6 & 6 & 6 \\
Number of females mated & 6 & 3 & 6 \\
Number of females pregnant & 6 & 3 & 0 \\
\% pregnancy & $100 \%$ & $50 \%$ & $0 \%$ \\
Gestational length & $23.30 \pm 00.33$ & $28 \pm 00.56^{*}$ & - \\
Total no. of pups born / rat & $(23-24$ days) & $(27-29$ days) & - \\
Pup viability & $(7-8)$ & $(1-4)$ & - \\
Sex ratio (M:F) & $100 \%$ & $80 \%$ & - \\
\hline
\end{tabular}

Values are Mean \pm SE; Significant difference at $(\mathrm{p}<0.05)$ control and treated rats. 
induced rats (Turner et al 2002). Treatment with deltamethrin $(50 \mathrm{mg} / \mathrm{kg}$ bw) also resulted in slight decrease in the mean pup weight (Wrenn et al., 1980). Reduced body weight gain of pups was observed in $1 / 10^{\text {th }}$ and $1 / 20^{\text {th }}$ of $\mathrm{LD}_{50}$ triazophos treated group during 30 days postnatal development (Sharma and Sangha, 2014). The maternal toxicity could be the trigger for the decreased pup weight gain (Farag et al., 2007). The undersized progeny might have developed alterations in neuromuscular parameters afterwards. It has been quoted that the higher level of sensitivity of the neonatal mammals to pyrethroid toxicity might be due to partial ripeness of the enzymes which catalyze the pyrethroids metabolism in the liver of juveniles (Farag et al., 2007).

Developmental and behavioural milestones: It was noticed that physical parameters were affected by ADI of deltamethrin treatment in rats progeny as compared to control rats progeny i.e. there was significant advancement in ear folding and also there was significant delay in incisor eruptions in ADI of deltamethrin treated rats progeny as compared to control rats progeny. Other parameters like pinna detachment, eye opening and fur development was effected non significantly in the pups born to treated rats (Table 3). Deltamethrin affected left eye of pup and it did not open in rat.

Sheet (2000) reported that deltamethrin treated young rats were considerably more sensitive then adult to lethal dose. Developmental landmarks such as development of fur, ear opening and eye opening was delayed in rats exposed to cyhalothrin throughout the pregnancy, (Gomes et al 1991 a,b). Husain and Seth (1991) reported that when deltamethrin was administered at $7 \mathrm{mg} / \mathrm{kg} /$ day to pregnant rats on gestation days 5 to 21 and to post-weaning rats on postnatal days 22 to 37 , the treated pups during gestation had delayed developmental landmarks. A significant delay in the day of eyes opening for both male and female offspring exposed maternally to $0.08 \mathrm{mg} / \mathrm{kg}$ deltamethrin was observed by Lazarini et al. (2001). Farag et al. (2007) commented that maternal toxicity could be the trigger for the delayed development of physical features in the high dose group (50\% of the control). Developmental milestones were nonsignificantly altered except fur development which was delayed in pups of $1 / 10^{\text {th }}$ and $1 / 20^{\text {th }}$ of $\mathrm{LD}_{50}$ triazophos treated group (Sharma and Sangha, 2014).

The study on the functional state of rat pup nervous system at different stages of postnatal development revealed differences in terms of when the reflex expressed. It was observed that control rats had taken more time for hanging as compared to treated rats and their grip became sharp on alternate days but treated pups took more days for sharp hanging grip (Table 4). It was also observed that control rats offsprings had sharp negative geotaxis and palmer grasp (at $25^{\circ}$ and $45^{\circ}$ ) as compared to treated rats offsprings, while some treated rats offsprings didn't showed negative geotaxis. Also, there was a significant difference in cliff avoidance in pups exposed to ADI of deltamethrin in utero as compared to control rats progeny. A gap was observed in hind limb and forelimb placement in treated rats progeny as compared to control rats progeny. Control rats pups were more active as compared to treated rats. Delayed onset of certain reflexes was also observed in rats exposed to deltamethrin at $7 \mathrm{mg} / \mathrm{kg} /$ day on gestation days 5 to 21(Husain and Seth, 1991). However, no gross abnormalities with the exception of a delay in the development of the surface-righting reflex was observed in rats exposed in utero to low-level deltamethrin (1 mg/kg bw/day) (Aziz et al 2001). Maternal and offspring body weight, physical and reflex development were found unaffected by the exposure to the $0.08 \mathrm{mg} /$ $\mathrm{kg}$ deltamethrin pesticide (Lazarini et al., 2001). Dams treated with $2.0 \mathrm{mg} / \mathrm{kg} /$ day $\mathrm{MeHg}$ (Methyl mercury) group had no significant effects on the ages of alter cliff avoidance, surface righting, startle reflex, pivoting, negative geotaxis, or forelimb and hindlimb grip strength in either sex (Gandhi et al., 2014).

\section{Conclusion}

The reproductive toxicity caused by deltamethrin lead to reduction in sexual competency, reproductive efficiency and fertility in animals. The ADI level of

Table 2. Changes in body weight gain (g/week) of pups born to control and treated rat mothers.

\begin{tabular}{llllllll}
\hline Treatments & $\mathbf{I}^{\text {st }}$ week & II $^{\text {nd }}$ week & III $^{\text {rd }}$ week & IV $^{\text {th }}$ week & $\mathbf{V}^{\text {th }}$ week & VI $^{\text {th }}$ week & VII $^{\text {th }}$ week \\
\hline Control & $14.06 \pm 0.26$ & $23.06 \pm 0.78$ & $33.80 \pm 0.99$ & $46.08 \pm 1.28$ & $59.80 \pm 1.74$ & $71.90 \pm 1.69$ & $84.05 \pm 1.58$ \\
ADI of & $8 \pm 1.30^{*}$ & $18.80 \pm 1.07^{*}$ & $31.20 \pm 0.74$ & $48.04 \pm 0.24$ & $61.80 \pm 2.39$ & $82.20 \pm 2.13$ & $100.60 \pm 2.62$ \\
Deltamethrin & & & & & & $*$ & $*$ \\
\hline
\end{tabular}

Values are Mean \pm SE; Significant difference at $(\mathrm{p}<0.05)$ control and treated rats.

Table 3. Physical development (in days) milestones in control and in utero exposed to ADI of deltamethrin pups.

\begin{tabular}{lccccc}
\hline Dose & $\begin{array}{c}\text { Pinna } \\
\text { detachment }\end{array}$ & $\begin{array}{c}\text { Incisor } \\
\text { eruptions }\end{array}$ & Fur development & Eye opening & Ear folding \\
\hline Control & $3 \pm 0.30$ & $7 \pm 0.00$ & $8 \pm 0.30$ & $14.00 \pm 0.508$ & $11 \pm 0.30$ \\
& $(2-3$ days $)$ & $(6-7$ days $)$ & $(8-9$ days $)$ & $(13-14$ days $)$ & $(10-11$ days $)$ \\
ADI of & $3.33 \pm 0.67$ & $6.33 \pm 3.17^{*}$ & $7.67 \pm 0.67$ & $13.30 \pm 0.33$ & $8 \pm 0.30^{*}$ \\
Deltamethrin & $(2-4$ days $)$ & $(6-9$ days $)$ & $(7-8$ days $)$ & $(13-14$ days $)$ & $(7-8$ days $)$ \\
\hline
\end{tabular}

Values are Mean \pm SE; Significant difference at $(\mathrm{p}<0.05)$ control and treated rats. 
Table 4. Effect of in utero exposure of deltamethrin on behavioural parameters (in days) in control and treated rats.

\begin{tabular}{lcc}
\hline Dose groups Tests & Control & ADI of deltamethrin \\
\hline Rightening reflex & $24.48 \pm 0.18$ & $23.79 \pm 0.27$ \\
Hanging grip test & $15.60 \pm 01.78$ & $19 \pm 02.63$ \\
Palmer grasp & $17.26 \pm 0.31$ & $17.02 \pm 0.30$ \\
Negative geotaxis & +++ & + \\
Cliff avoidance & $10.80 \pm 0.20$ & $09.80 \pm 0.37^{*}$ \\
\hline
\end{tabular}

Values are Mean \pm SE; Significant difference at $(\mathrm{p}<0.05)$ control and treated rats; $+=$ mild presence, $+++=$ strong presence

deltamethrin exposure during pregnancy affected the litter size, growth development and behavior of offsprings while 10 times ADI level of deltamethrin exposure during pregnancy induced post implantation losses in rats. The present study, thus, shows a great deal of variability in the toxicity of deltamethrin at ADI and 10 times ADI levels.

\section{ACKNOWLEDGEMENTS}

Authors are thankful to Department of Zoology, PAU Ludhiana, Punjab (India) for providing necessary facilities and infrastructure to carry out the research.

\section{REFERENCES}

Abdel-Khalik, M. M., Hanafy, M. S. and Abdel-Aziz, M.I. (1993). Studies on the teratogenic effects of deltamethrin in rats. Deutsche Tierarztliche Wochenschrift, 100 (4):142-143.

Ahmad, L. (2010). Pathological effects of cypermethrin in rabbits. Ph.D dissertation. Department of Pathology, University of Agriculture Faisalabad, Pakistan.

Ahmad, L. and Khan, A. (2012). Pyrethroid-induced reproductive toxico-pathology in non-target species. Pak. Vet. J., 32: 1-9.

Ahmed N and Gupta P. K. (1988). Reproduction (one generation) toxicity of cypermethrin in rats. J. Environ. Biol., 9: 149-56.

Altman, J. and Sudarshan, K. (1975). Postnatal development of locomotion in the laboratory rat. Anim. Behav., 23: 896-920.

Aziz, M. H., Agrawal, A.K., Adhami, V. M., Shukla, Y. and Seth, P. K. (2001). Neurodevelopmental consequences of gestational exposure (GD14-GD20) to low dose deltamethrin in rats. Neurosci. Letters, 300 (3):161-165.

Berkowitz, G. S., Obel, J., Deych, E., Lapinski, R., Godbold, J., Liu, Z., Landrigan, P. J. and Wolff, M. S. (2003) Exposure to indoor pesticides during pregnancy in a multiethnic, urban cohort. Environ. Hlth. Perspect, 111: 79-84.

Bhaumik, A. and Gupta, P. K. (1990). Teratogenicity of deltamethrin in rats. Indian Vet. J., 67 (3): 213-219.

Biernacki, B., Wlodarczyk, B., Minta, M. and Juszkiewicz, T. (1995). Influence of cypermethrin on pregnancy and fetal development in rabbits. Med. Wet., 51: 31-33.

Eskenazi, B., Harley, K., Bradman, A., Weltzien, E., Jewell, N. P and Barr, D. B. (2004). Association of in utero organophosphate pesticide exposure and fetal growth and length of gestation in an agricultural population. Environ. Hlth. Perspect., 112: 1116-1124.

Farag, A.T., Goda, N.F., Shaaban, N.A. and Mansee, A.H. (2007). Effects of oral exposure of synthetic pyrethroid, cypermethrin on the behavior of F1-progeny in mice.
Reprod. Toxicol., 23: 560-567.

Gandhi, D.N., Panchal, G.M. and Dhull, D.K. (2014). Neurobehavioral toxicity in progeny of rat mothers exposed to methylmercury during gestation. Annali dell'Istituto Superiore di Sanità., 50: 28-37.

Garry, V.V. (2004). Pesticides and children. Toxicol Appl Pharmacol., 198 (2): 152-163

Gomes, M.S., Bernardi, M.M. and Spinosa, H.S. (1991a). Effects of prenatal pyrethroid insecticide exposure on the sexual development of rats. Vet. Hum. Toxicol. 33: 427-428.

Gomes, M.S., Bernardi, M.M. and Spinosa, H.S. (1991b). Pyrethroid insecticides and pregnancy: effect on physical and behavioural development of rats. Vet. Hum. Toxicol. 33: 315-317.

Huang, C. and Li, X. (2014). Maternal Cypermethrin |Exposure during the Perinatal Period Impairs Testicular Development in C57BL Male Offspring. PLoS ONE. 9 (5): 96781.

Husain, R. and Seth, P.K. (1991). Neurotoxic effects of deltamethrin, a synthetic pyrethroid during early development in rats. Int. J. Toxicol. Occup. Env. Hlth., 1(1): 138

James (2008). Evidence that mammalian sex ratios at birth are partially controlled by parental hormone levels around the time of conception. J. Endocrinol., 198: 315.

Kandil, A.M. (2006). Toxic Effects of Deltamethrin on the Pregnant Rats and Their Fetuses. J. Drug. Res., 27: 8289.

Kumar, A. and Nagar, M. (2014). Histomorphometric study of testis in deltamethrin treated albino rats. Toxicology Reports., 1: 401-410.

Lapointe, G. and Nosal, G. (1979). A rat model of neurobehavioral development. Experientia Basel., 35: 205-207.

Lazarini, C.A., Florio, J.C., Lemonica, I.P. and Bernardi, M.M. (2001). Effects of prenatal exposure to deltamethrin on forced swimming behavior, motor activity, and striatal dopamine levels in male and female rats. Neurotoxicol Teratol., 23 (6): 665-673.

Lemos, A.J.J.M., Wanderley-Teixeira, V., Teixeira, A.A.C., FdCA, Silva., Oliveira, J.V. and de Siqueira, H.A.A. (2011). Response of Blastocyst-Endometrium Interactions in Albino Rats to Sublethal Doses of Biological and Synthetic Insecticides. Food Chem. Toxicol., 49: 25412547.

Li, X. and Rehman, N. (2008) Impact of androgen/estrogen ratio: lessons learned from the aromatase over expression mice. Gen. Comp. Endocrinol., 159: 1-9.

Li, X., Nokkala, E., Yan, W., Streng, T. and Saarinen, N. (2001). Altered structure and function of reproductive organs in transgenic male mice overexpressing human aromatase. Endocrinol., 142: 2435-2442.

McGregor, D.B. (2000). Pesticide residues in Food. 
Deltamethrin International agency for research on cancer. Lyon, France.

McLachlan, J.A. (2001). Environmental signaling: what embryos and evolution teach us about endocrine disrupting chemicals? Endo. Rev., 22: 319-341.

Mocarelli, P., Gerthoux, P.M., Ferrari, E., Patterson, D.G. and Kieszak, S.M. (2000). Paternal concentrations of dioxin and sex ratio of offspring. Lancet., 355: 18581863.

Muthviveganandavel, V., Muthuraman, P., Muthu, S. and Srikumar, K. (2008). A study on low dose cypermethrin induced histopathology, lipid peroxidation and marker enzyme changes in male rat. Pesticide Biochem Physiol., 9:12-16.

Presibella, K.M., Kita, D.H., Carneiro, C.B., Andrade, A.J. and Dalsenter, P.R. (2005). Reproductive evaluation of two pesticides combined (deltamethrin and endosulfan) in female rats. Reprod. Toxicol., 20 (1): 95-101.

Schettgen, T., Koch, H.M., Drexler, H. and Angerer, J. (2002). Newgaschromatographic- mass spectrometric method for the determination of urinary pyrethroid metabolites in environmental medicine. J. Chromatogr. B .Analyt. Technol. Biomed. Life Sci., 778: 121-130.

Sharma, D. and Sangha, G.K. (2014). Triazophos induced oxidative stress and histomorphological changes in liver and kidney of female albino rats. Pest Biochem. Physiol., 110:71-80.

Sheet, L.P. (2000). A consideration of age-dependent differences in susceptibility to organophosphorus and pyrethroid insecticides. Neurotoxicol., 21: 57-63.

Shukla, Y. and Taneja, P. (2002). Mutagenic potential of cypermethrin in mouse dominant lethal assay. J. Environ. Pathol. Toxicol. Oncol., 21: 259-265.

Turner, K.J., Barlow, N.J., Struv., M.F., Wallance, D.G., Gaido, K.W., Dorman, D.C. and Foster, D.M.D. (2002). Effect of in utero exposure to the organophosphate insecticide Fenintrothion on Androgen- Dependent Reproductive development in the Crl: $\mathrm{CD}(\mathrm{SD}) \mathrm{BR}$ Rat. Toxicological Sci., 68: 17.

Ullah, M.S., Ahmad, M., Ahmad, N., Khan, M.Z. and Ahmad, I. (2006). Toxic effects of cypermethrin in female rabbits. Pak. Vet. J., 26: 193-196.

Wigle, D.T., Arbuckle, T.E., Turner, M.C., Bérubé, A. and Yang, Q. (2008). Epidemiologic evidence of relationships between reproductive and child health outcomes and environmental chemical contaminants. J. Toxicol. Environ. Hlth. B. Crit. Rev., 11: 373-317.

Wrenn, J.M., Rodwell, D.E., Goldenthal, E.I., Spider, E.J.C. and Rajasekaran, D. (1980). Three generation reproduction study in rats. CDPR. Mattawan, IRDC Study.

Xavier, R., Rekha, K. and Bairy and K.L. (2004). Health perspective of pesticide exposure and dietary management. Mal. J. Nutr., 10: 39-51.

Yousef, M.I., El-Demerdash. and Al-Salhen, K.S. (2003). Protective role of isoflavones against the toxic effect of cypermethrin on semen quality and testosterone levels of rabbits (Anglais, abstract in English). J. Environ. Sc. Hlth. B., 38: 463-478.

Zober, A., Hoffmann, G., Ott, M.G., Will, W. and Germann, C. (1995). Study of morbidity of personnel with potential exposure to vinclozolin. Occup. Environ. Med., 52: $233-$ 241. 